\title{
Original Article Markers of Oxidative Stress in the Saliva of Type 2 Diabetic Patients
}

\author{
Olatunde Olayanju ${ }^{1,2}$, Victory Fabian Edem ${ }^{3}$, Chika Okwor ${ }^{4}$, Gabriel Odok ${ }^{1}$, Nnaemeka Awah ${ }^{1}$ \\ ${ }^{1}$ Chemical Pathology Department, University College hospital, Ibadan, Nigeria., \\ ${ }^{2}$ Chemical Pathology Department, Babcock University Teaching Hospital, Ilisan, Nigeria, \\ ${ }^{3}$ Immunology Department, College of Medicine, University of Ibadan, Nigeria, \\ ${ }^{4}$ Chemical Pathology Department, University of Nigeria Teaching Hospital Ituku/Ozalla, Enugu, Nigeria. \\ *Corresponding Author: olayanjuo@babcock.edu.ng
}

Received: $19-7-2021$

Revised: 3-9-2021

Published: 22-9-2021

\section{Keywords:}

Diabetes mellitus,

Saliva,

Hydrogen peroxide,

Nitric oxide,

Malondialdehyde

\begin{abstract}
Objective: Diabetes mellitus is associated with increased prevalence of oral diseases for which reactive oxygen species have been implicated. The saliva contains protective antioxidants which statutorily curtail these destructive oxygen molecules. A functional compromise of the antioxidants may precipitate oxidative stress leading to the increased oral disease susceptibility. However, salivary markers of oxidative stress have not been sufficiently studied in the diabetics. Methods: A total of 166 adults were recruited for this study. They comprised of 95 Type 2 diabetic patients and 71 healthy non-diabetic controls. About $3 \mathrm{ml}$ of unstimulated saliva samples were collected from participants and processed, levels of salivary $\mathrm{H}_{2} \mathrm{O}_{2}, \mathrm{NO}$ and MDA were measured using spectrophotometry method and compared between the two groups. Data was analysed using ttest, logistic regression and receiver operating characteristics (ROC) with statistical significance set at $\mathrm{p}<0.05$. Results: Salivary $\mathrm{H}_{2} \mathrm{O}_{2}(\mathrm{p}=0.024)$ and $\mathrm{NO}(\mathrm{p}=0.002)$ were significantly higher in the diabetic patients when compared to the healthy non-diabetic control group. Binary logistic regression showed that patients with Type 2 diabetic mellitus are more likely to have elevated salivary $\mathrm{H}_{2} \mathrm{O}_{2}(\mathrm{OR}=1.013 ; \mathrm{p}=0.025)$ and $\mathrm{NO}(\mathrm{OR}=1.016 ; \mathrm{p}=0.003)$ levels. $\mathrm{ROC}$ analysis showed statistically significant performance of salivary NO levels in distinguishing between T2DM patients and healthy controls. Conclusions: Higher levels of oxidative stress markers including salivary $\mathrm{H}_{2} \mathrm{O}_{2}$ and $\mathrm{NO}$ in the diabetic groups could be a pointer to the characteristic high prevalence of oral diseases in diabetes mellitus, given that oxidative stress predisposes to disease vulnerability. This calls for increased attention to oral health in diabetes management to minimise co-morbidity.
\end{abstract}

Cite this article as: Olayanju, O., Edem, VF., Okwor, C., Odok, G. and Awah, N. (2021). Markers of Oxidative Stress in the Saliva of Type 2 Diabetic Patients. Journal of basic and applied Research in Biomedicine, 7(2): 1-4 https://doi.org/10.51152/jbarbiomed.v7i2.217

CC 1 (1) $\begin{aligned} & \text { This work is licensed under a Creative Commons Attribution } 4.0 \text { License. You are free to copy, distribute and perform the work. You must attribute } \\ & \text { the work in the manner specified by the author or licensor. }\end{aligned}$

\section{INTRODUCTION}

The oral mucosa is one of the key body sites with major pathologic sequalae of diabetes mellitus (DM). Clinical conditions including halitosis, lichen planus, gingivitis, periodontitis, angular cheilitis etc. occur more frequently in diabetic patients compared to the general population (AlMaskari, Al-Maskari, \& Al-Sudairy, 2011). Although DM which is a chronic disorder of intermediary metabolism, characterised by chronic hyperglycaemia, is associated with generalised immune dysfunction (Berbudi, Rahmadika, Cahyadi, \& Ruslami, 2019), several studies have equally implicated reactive oxygen species in both the pathogenesis of the disease and the development of complications including oral diseases (Maritim, Sanders, \& Watkins, 2003). These free radicals which result from glucose oxidation, non-enzymatic glycation of plasma proteins and subsequent degradation of such proteins, are injurious to cells, organelles and lipid-rich cell membranes thereby predisposing to the increased inflammatory and infective diseases in the oral cavity (Maritim et al., 2003).

Furthermore, DM is associated with grossly depleted levels of antioxidant enzymes including catalase, glutathione peroxidase, superoxide dismutase and water-soluble vitamins (Trivedi et al., 2014). These agents which primarily function as free radical scavengers are consumed at a higher rate than they are produced in affected patients (Bajaj \& Khan, 2012). Thus, low levels encourage the proliferation of reactive oxygen species which are very toxic to cells either directly or through their metabolites (Demircan et al., 2008; Rehman \& Akash, 2017). Hydrogen peroxide $\left(\mathrm{H}_{2} \mathrm{O}_{2}\right)$, for example, causes irreparable damages to DNA, RNA and lipids (Asmat, Abad, \& Ismail, 2016). Also, nitric oxide (NO) which plays a vital role in the relaxation of blood vessels causes vascular injuries in DM because of excessive production (Desmond Jay, Hirofumi Hitomi, \& Griendling, 2006; Hoang, Padgham, \& Meininger, 2013; Pitocco, Tesauro, Alessandro, Ghirlanda, \& Cardillo, 2013; Pitocco et al., 2010). Consequently, there is a significant elevation of malondialdehyde (MDA), a prominent product of lipid peroxidation, and this is a common finding in diabetes mellitus (Nakhjavani et al., 2010; Slatter, C.H. Bolton, \& Bailey, 2000; Zygula et al., 2019).

Most of the available diabetes studies interrogating the role of oxidative stress in systemic infections were done in the serum (Bhutia, Ghosh, Sherpa, Pal, \& Mohanta, 2011; Blasiak et al., 2004; Gallou et al., 1993). The saliva, which is the main fluid of the oral cavity and has a rich antioxidant capacity, has hardly been sufficiently studied in the diabetics. The saliva has however proven to be resourceful in providing useful information regarding systemic disease. Salivary antioxidant status and lipid peroxidation have been studied in cigarette smokers to determine their contribution to the development of periodontitis (Kosoko, Olayanju, Rahamon, \& Arinola, 2017); salivary cortisol have been used to screen, diagnose and monitor patients with Cushing' s disease (Bozovic, Racic, \& Ivkovic, 2013; Papanicolaou, Mullen, Kyrou, \& Nieman, 2002); and salivary markers of oxidative stress have been studied in several other conditions like ageing, dementia, and acute asthma (Bentur, Mansour, Brik, Eizenberg, \& Nagler, 2006; Choromanska et al., 2017; Maciejczyk, Zalewska, \& Ladny, 2019). In this study, we aimed to measure salivary $\mathrm{H}_{2} \mathrm{O}_{2}$, NO and MDA in diabetic patients in comparison to healthy nondiabetic controls.

\section{MATERIALS AND METHODS \\ Participants}

This was a cross-sectional study involving adult Type 2 diabetic patients and healthy non-diabetic control group. The diabetic patients were recruited from the medical outpatient clinic of the University College Hospital, Ibadan, while healthy non-diabetic members of staff served as the control group. A questionnaire was used to obtain demographic and clinical information from the patients and anthropometric measurements including weight and height were taken of all participants. Figure 1 shows the 
flow diagram for diabetic patients' recruitment. Ethical approval was obtained from the University of Ibadan/ University College Hospital Research Ethics Committee.

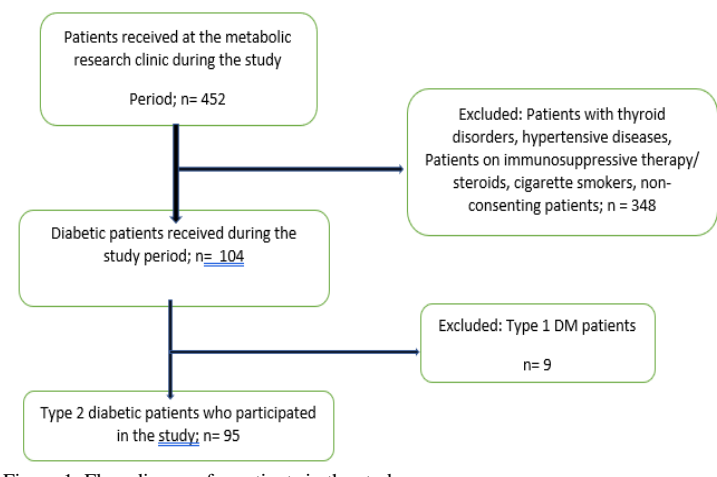

Figure 1: Flow diagram for patients in the study

\section{Sample Collection}

About $3 \mathrm{ml}$ of unstimulated saliva samples were collected from all participants after rinsing their mouth with clean water, samples were collected passively in a clean universal bottle by making the participants drool. The samples were centrifuged at a speed of 3000 radian per minute for five minutes, the supernatant transferred to clean Eppendorf bottles and stored at $20{ }^{\circ} \mathrm{C}$ until the time of analysis. Levels of $\mathrm{H}_{2} \mathrm{O}_{2}$, NO and MDA were measured in all saliva samples. About $3 \mathrm{ml}$ of blood samples were also drawn from all participants into a fluoride oxalate bottle for measuring fasting plasma glucose in the diabetic patients and random plasma glucose in the healthy nondiabetic participants. Samples were centrifuged at a speed of 4000 radian per minute for five minutes, the plasma transferred into a clean plain bottle and stored at $-20^{\circ} \mathrm{C}$ until the time of analysis. The study lasted for three months.

\section{Laboratory Analysis}

Malondialdehyde was estimated using the thiobarbituric acid reactive substance (TBARS) test (Bernheim et al., 1947). In this assay, free MDA present in the sample reacts with Thiobarbituric Acid (TBA) and generates MDA-TBA adduct with absorbance maximum at $532 \mathrm{~nm}$ wavelength. Salivary MDA was determined by comparing absorbance with MDA standard.

Hydrogen peroxide concentration was determined as described by Wolff (1994). The assay is based on peroxide-mediated oxidation of $\mathrm{Fe}^{2+}$, followed by the reaction of $\mathrm{Fe}^{3+}$ with xylenol orange to form $\mathrm{Fe}^{3+}$-xylenol orange complex with absorbance maximum at $560 \mathrm{~nm}$ wavelength. Salivary $\mathrm{H}_{2} \mathrm{O}_{2}$ was determined by comparing absorbance with standard solutions of $\mathrm{H}_{2} \mathrm{O}_{2}$.

Nitric oxide concentration in saliva was determined using Griess reagent (Sulpanilamide and N-1-napthyethylenediamine dihydrochloride). The assay is based on a reaction that utilizes sulpanilamide and $\mathrm{N}$-1-napthyethylenediamine dihydrochloride (NED) under acidic (phosphoric acid) conditions. Nitrite forms coloured chromophore with reagent, with absorbance maximum at $540 \mathrm{~nm}$ wavelength. The production of nitrite was quantified by comparing the result with absorbances of standard solutions of sodium nitrite.

Plasma glucose levels in both the patients and the control group were determined using the glucose oxidase method. The assay is based on enzymatic oxidation of glucose into gluconic acid and hydrogen peroxide, which is by a chromogenic oxygen acceptor in the presence of a peroxidase. Glucose concentration is determined by relating the optical density of sample at $500 \mathrm{~nm}$ to that of a glucose standard.

\section{Statistical Analysis}

The effect of the reactive oxygen species was determined by comparative analysis of data between the diabetic patients and healthy non-diabetic adults. Data was summarized as Mean \pm SEM. Mean comparisons between cases and controls were carried out using Student t-test and test of associations were carried out using binary logistic regression. Area under curve, and best estimates of specificity and sensitivity were evaluated with the Receiver Operator Characteristics (ROC) curve. Statistical significance was set at $\mathrm{p} \leq 0.05$. Data obtained were analysed using statistical package for social sciences (SPSS) version 20.0 (IBM Inc., USA).

\section{RESULTS}

A total of one hundred and sixty-six participants were recruited for this study. While 95 (57.2\%) of them were known diabetic patients on treatment, the remaining $71(42.8 \%)$ participant were healthy non-diabetic adults. There were no significant differences in the age and weight between the diabetic and the healthy controls, though the BMI was significantly higher in the diabetic patients.

There was no significant different in the levels of salivary MDA between the two groups $(\mathrm{p}=0.845)$, however salivary $\mathrm{H}_{2} \mathrm{O}_{2}$ $(\mathrm{p}=0.024)$ and $\mathrm{NO}(\mathrm{p}=0.002)$ were significantly higher in the diabetic patients compared to the healthy non-diabetic control group (Table 1).

Table 1: Comparison of mean age, anthropometric parameters, salivary oxidants and MDA levels in Type 2 diabetes patients and non-diabetic controls

\begin{tabular}{|c|c|c|c|c|}
\hline Variable & Cases (n=95) & Control (n=71) & $\mathrm{t}$ & $\mathbf{P}$ \\
\hline Age & $58.89 \pm 1.41$ & $55.48 \pm 1.10$ & 1.793 & 0.075 \\
\hline Gender (Male) & $31(32.6)^{\mathrm{a}}$ & $49(71.0)^{\mathrm{a}}$ & b23.568 & $0.000^{*}$ \\
\hline Body weight & $73.99 \pm 1.76$ & $71.84 \pm 1.58$ & 0.895 & 0.372 \\
\hline Height & $1.59 \pm 0.01$ & $1.67 \pm 0.01$ & 4.926 & $0.000^{*}$ \\
\hline BMI & $29.20 \pm 0.67$ & $26.03 \pm 0.74$ & 3.092 & $0.002 *$ \\
\hline Salivary MDA & $1.14 \pm 0.05$ & $1.13 \pm 0.05$ & 0.164 & 0.845 \\
\hline Salivary $\mathrm{H}_{2} \mathrm{O}_{2}$ & $62.90 \pm 3.54$ & $52.03 \pm 2.81$ & 2.282 & $0.024 *$ \\
\hline Salivary NO & $42.13 \pm 4.43$ & $24.18 \pm 2.73$ & 3.181 & $0.002 *$ \\
\hline
\end{tabular}

Binary logistic regression showed that patients with Type 2 diabetic mellitus are more likely to have elevated salivary $\mathrm{H}_{2} \mathrm{O}_{2}$ $(\mathrm{OR}=1.013 ; \mathrm{p}=0.025)$ and $\mathrm{NO}(\mathrm{OR}=1.016 ; \mathrm{p}=0.003)$ levels (Table 2). ROC analysis showed statistically significant performance of salivary NO levels in distinguishing between T2DM patients and controls with AUC of 0.633 , sensitivity and specificity of $63.2 \%$ and $50.7 \%$ respectively (Table 3; Figure 2). There were no significant associations between salivary MDA, $\mathrm{H}_{2} \mathrm{O}_{2}$ and $\mathrm{NO}$ levels with fasting plasma glucose level, duration of T2DM as well as comorbidity in T2DM patients (Tables 4).

Table 2: Binary logistic regression analysis of association between Type 2 Diabetes Mellitus (T2DM) and salivary oxidants and MDA level

Mellitus (T2DM) and salivary oxidants and MDA levels
\begin{tabular}{|l|l|l|l|l|}
\hline Variable & B & P & Exp $\boldsymbol{\beta}$ & 95\% CI \\
\hline Salivary MDA & -0.140 & 0.720 & 0.869 & $0.404-1.870$ \\
\hline Salivary $\mathbf{H}_{2} \mathbf{O}_{2}$ & 0.013 & $0.025 *$ & 1.013 & $1.002-1.025$ \\
\hline Salivary NO & 0.016 & $0.003 *$ & 1.016 & $1.006-1.027$ \\
\hline
\end{tabular}
*Significant at p $<0.05$

*Significant at $\mathrm{p}<0.05$

Table 3: ROC Analysis of salivary MDA, $\mathrm{H}_{2} \mathrm{O}_{2}$ and NO levels

\begin{tabular}{|l|l|l|l|l|l|}
\hline Variable & AUC & \multicolumn{1}{l}{$\mathbf{9 5 \%}$ CI } & \multicolumn{1}{c|}{ p } & \multicolumn{1}{c|}{ Sensitivity } & Specificity \\
\hline Salivary MDA & 0.507 & $0.418-0.596$ & 0.879 & 50.5 & 54.9 \\
\hline Salivary $\mathbf{H}_{2} \mathbf{O}_{2}$ & 0.588 & $0.500-0.676$ & 0.052 & 51.6 & 49.3 \\
\hline Salivary NO & 0.633 & $0.548-0.719$ & $0.003^{*}$ & 63.2 & 50.7 \\
\hline
\end{tabular}

*Significant at $\mathrm{p}<0.05$

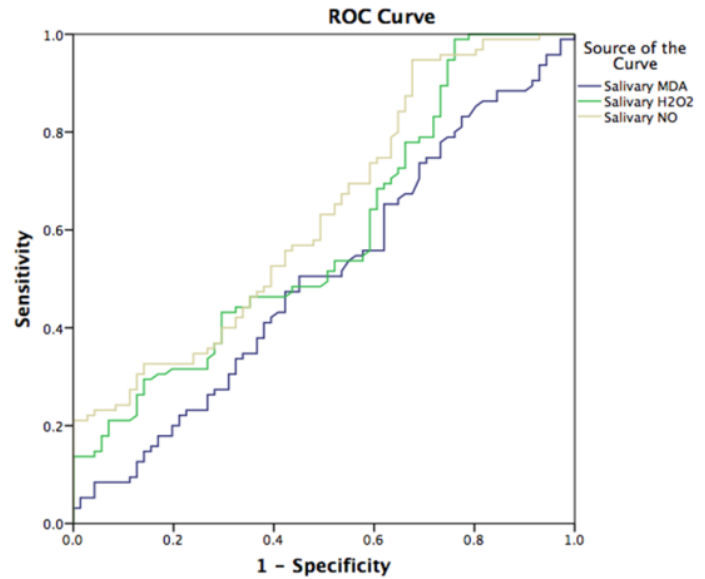

Figure 2: Receiver operating characteristics (ROC) curve of salivary MDA, $\mathrm{H}_{2} \mathrm{O}_{2}$ and NO levels 
Table 4: Binary logistic regression analysis of association between fasting plasma glucose level, DM duration and Co-morbid condition, and salivary oxidants and MDA levels among Type 2 Diabetes Mellitus (T2DM) patients

\begin{tabular}{|c|c|c|c|c|}
\hline Variable & B & $\mathbf{p}$ & $\operatorname{Exp} \beta$ & $95 \% \mathrm{CI}$ \\
\hline \multicolumn{5}{|c|}{ Fasting plasma glucose } \\
\hline $\begin{array}{l}\text { Salivary } \\
\text { MDA }\end{array}$ & -1.058 & 0.139 & 0.347 & $0.085-1.411$ \\
\hline $\begin{array}{l}\text { Salivary } \\
\mathrm{H}_{2} \mathrm{O}_{2}\end{array}$ & -0.002 & 0.805 & 0.998 & $0.982-1.014$ \\
\hline Salivary NO & 0.010 & 0.110 & 1.010 & $0.998-1.022$ \\
\hline \multicolumn{5}{|l|}{ DM duration } \\
\hline $\begin{array}{l}\text { Salivary } \\
\text { MDA }\end{array}$ & -0.122 & 0.826 & 0.885 & $0.297-2.634$ \\
\hline $\begin{array}{l}\text { Salivary } \\
\mathrm{H}_{2} \mathrm{O}_{2}\end{array}$ & -0.005 & 0.515 & 0.995 & $0.981-1.009$ \\
\hline Salivary NO & 0.007 & 0.161 & 1.007 & $0.997-1.018$ \\
\hline \multicolumn{5}{|c|}{ Co-morbid condition } \\
\hline $\begin{array}{l}\text { Salivary } \\
\text { MDA }\end{array}$ & $\begin{array}{c}-0.013 \\
\end{array}$ & 0.980 & 0.987 & $0.370-2.634$ \\
\hline $\begin{array}{l}\text { Salivary } \\
\mathrm{H}_{2} \mathrm{O}_{2}\end{array}$ & 0.003 & 0.643 & 1.003 & $0.991-1.015$ \\
\hline Salivary NO & -0.005 & 0.350 & 0.995 & $0.985-1.005$ \\
\hline
\end{tabular}

\section{DISCUSSION}

Diabetes mellitus is associated with increased prevalence of oral disease, and reactive oxygen species have been implicated in their pathogenesis (Indurkar, Maurya, \& Indurkar, 2016; Kadir et al., 2002). In this study, we found significantly higher salivary levels of $\mathrm{H}_{2} \mathrm{O}_{2}$ in diabetic patients compared to the controls. This agrees with the reports of Leoncini et al (Leoncini, Signorello, Piana, Carrubba, \& U, 1997) and Awatef et al (Awatef, Abdelh, \& Asma, 2013), who found higher levels of $\mathrm{H}_{2} \mathrm{O}_{2}$ using blood samples from diabetic patients compared to control groups. The impaired secretion of antioxidants in the diabetics have been implicated in the uncontrolled production of $\mathrm{H}_{2} \mathrm{O}_{2}$ and its deleterious effects (Thomas, Ramesh, Suresh, \& Prasad, 2013; Trivedi et al., 2014). This could explain increased prevalence of oral disorders in patients with diabetes mellitus and supports the need for increased awareness of the associations between diabetes and oral health.

Elevated salivary NO levels was implicated in the development of mucosa erosion and ulceration (Sunitha \& Shanmugam, 2006). In this study, we found significantly higher levels of salivary NO in the diabetics compared to the healthy nondiabetic controls. This is similar to findings in a previous study where elevated NO was found in plasma and saliva of diabetic patients (Astaneie et al., 2005). The increased secretion was attributed to a reactive response to hyperglycaemia, a cardinal fulcrum in diabetes mellitus (Ceriello et al., 2002; Cosentino, Hishikawa, Katusic, \& Lüscher, 1997). Furthermore, due to the inflammatory sequela associated with the etiology of diabetes mellitus, increased salivary NO levels observed in diabetes patients could be indicative of ongoing inflammation in the oral cavity. Although excessive salivary NO has been previously implicated as a physio-pathological regulator in modifying oral mucosal disease, we posit that inflammation within the oral cavity could be the underlying mechanism that links salivary NO with oral mucosal disease.

This study demonstrated a significant association between diabetes mellitus and salivary $\mathrm{H}_{2} \mathrm{O}_{2}$ level $(\mathrm{OR}=0.013$; $\mathrm{p}=0.025)$ and salivary NO level $(\mathrm{OR}=0.016 ; \mathrm{p}=0.003)$, but not salivary MDA $(O R=-0.140 ; p=0.720)$, although ROC analysis indicated a statistically significant area under curve (AUC) for salivary NO with sensitivity and specificity of $63.2 \%$ and $50.7 \%$ respectively. This indicates that salivary $\mathrm{NO}$ would be a better marker than salivary $\mathrm{H}_{2} \mathrm{O}_{2}$ for distinguishing between diabetes patients and non-diabetic controls. It has also been suggested to be a good predictor of xerostomia in diabetic patients (Abadi, Koopaie, \& Montazeri, 2020). The low specificity of salivary NO observed in this study may be due to varying degrees of oral health in the participants which may have confounded the result. Increased NO synthesis is a frequent finding in periodontal diseases, radicular cysts and apical infections (Paquette \& Williams, 2000; Takeichi et al., 1999). Elevated levels have also been reported in periodontitis, benign diseases and malignant tumours of the salivary glands, including squamous cell carcinoma(Bentz, Haines, Hanson, \& Radosevich, 1998; Daghigh, Borghaei, Thornton, \& Bee, 2002). Further studies of salivary NO levels in diabetic patients with predetermined oral health status would make for better understanding of the relationship between diabetes mellitus and salivary NO.

There were no significant associations between salivary $\mathrm{H}_{2} \mathrm{O}_{2}$, $\mathrm{NO}$ and MDA levels with fasting plasma glucose level, duration of T2DM and comorbidity in T2DM patients in this study. This agrees with the study of Abadi et al. who reported no significant association between salivary NO level and blood glucose and HbA1c (Abadi et al., 2020). Although this indicates that salivary levels of $\mathrm{H}_{2} \mathrm{O}_{2}$, NO and MDA may not be directly influenced by degree of hyperglycaemia, duration of illness or presence of comorbidities. It could also be because of anti-diabetic therapy or lifestyle modifications adopted by diabetes patients.

There are some limitations to this study. Salivary levels of antioxidant markers should have given more credence to this study; however, several studies have shown that serum levels are reduced in diabetes (Trivedi et al., 2014). Serum HbA1c which is a marker of long-term glycaemic control would have also been evaluated rather than fasting plasma glucose to determine how the salivary markers changed with glycaemic control.

\section{CONCLUSION}

This study showed that the levels of salivary $\mathrm{H}_{2} \mathrm{O}_{2}$ and NO were significantly higher in diabetes patients than non-diabetic controls and could be a pointer to the high prevalence of oral diseases in diabetes. There is a need for increased awareness of the associations between diabetes and oral health with integration of oral health management into the management of diabetes mellitus given the associations between salivary levels of $\mathrm{H}_{2} \mathrm{O}_{2}$ and $\mathrm{NO}$ with oral mucosa diseases.

\section{Funding}

This study did not receive any funding or material support Conflict of interest

Authors have no conflict of interest to declare

\section{REFERENCES}

Abadi, P. A., Koopaie, M., \& Montazeri, R. (2020). Comparison of salivary nitric oxide and oral health in diabetic patients with and without xerostomia. Diabetes \& Metabolic Syndrome: Clinical Research \& Reviews, 14, 11-15.

Al-Maskari, A. Y., Al-Maskari, M. Y., \& Al-Sudairy, S. (2011). Oral manifestations and complications of diabetes mellitus: a review. Sultan Qaboos University Medical Journal, 11(2), 179.

Asmat, U., Abad, K., \& Ismail, K. (2016). Diabetes mellitus and oxidative stress-A concise review. Saudi Pharmaceutical Journal, 24(5), 547-553.

Astaneie, F., Afshari, M., Mojtahedi, A., Mostafalou, S., Zamani, M. J., Larijani, B., \& Abdollahi, M. (2005). Total Antioxidant Capacity and Levels of Epidermal Growth Factor and Nitric Oxide in Blood and Saliva of Insulin-Dependent Diabetic Patients. Arch Med Res, 36(4), 376- 381 .

Awatef, M., Abdelh, È., \& Asma, K. (2013). Hydrogen peroxide: An oxidant stress indicator in type 2 diabetes. $B C A I J, 7(4), 166-172$.

Bajaj, S., \& Khan, A. (2012). Antioxidants and diabetes Indian J of Endocrinol Metab, Dec; 16 (Suppl 2): S267-S271.

Bentur, L., Mansour, Y., Brik, R., Eizenberg, Y., \& Nagler, R. M. (2006). Salivary oxidative stress in children during acute asthmatic attack and during remission. Respiratory Medicine, 100, 1195- 1201.

Bentz, B. G., Haines, G. K. I., Hanson, D. G., \& Radosevich, J. A. (1998). Endothelial constitutive nitric oxide synthase (ecNOS) localization in normal and neoplastic salivary tissue. Head Neck, 20(4), 304-309.

Berbudi, A., Rahmadika, N., Cahyadi, A., \& Ruslami, R. (2019). Type 2 diabetes and its impact on the immune system [published ahead of print, 2019 Oct 23]. Curr Diabetes Rev 2019;10.2174/15733998156661910240858.

Bhutia, Y., Ghosh, A., Sherpa, M. L., Pal, R., \& Mohanta, P. K. (2011). Serum malondialdehyde level: Surrogate stress marker in the Sikkimese diabetics. Journal of natural science, biology, and medicine, 2(1), 107. 
Blasiak, J., Arabski, M., Krupa, R., Wozniak, K., Zadrozny, M., Kasznicki, J., . . . Drzewoski, J. (2004). DNA damage and repair in type 2 diabetes mellitus. Mutation Research/Fundamental and Molecular Mechanisms of Mutagenesis, 554(1-2), 297-304.

Bozovic, D., Racic, M., \& Ivkovic, N. (2013). Salivary cortisol levels as a biological marker of stress reaction. Med Arch, 67(5), 374- 377

Ceriello, A., Quagliaro, L., Catone, B., Pascon, R., Piazzola, M., Bais, B., . . Motz, E. (2002). Role of Hyperglycemia in Nitrotyrosine Postprandial Generation. Diabetes care, 25(8), 1439-1443.

Choromanska, M., Klimiuk, A., Kostecka-Sochon, P., Wilczynska, K., Kwiatkowski, M., Okuniewska, N., . . . Maciejczyk, M. (2017). Antioxidant defence, oxidtive stress, and oxidative damage in saliva,plasma and erythrocyte of dementia patients. Can Salivary AGE be a marker of Dementia? Int J Mol Sci, 18(10).

Cosentino, F., Hishikawa, K., Katusic, Z. S., \& Lüscher, T. F. (1997). High glucose in-creases nitric oxide synthase expression and superoxide anion generation in humanaortic endothelial cells. Circulation, 96, 25- 28.

Daghigh, F., Borghaei, R. C., Thornton, R. D., \& Bee, J. H. (2002). Human gingival fibroblasts produce nitric oxide in response to proinflammatory cytokines. . $J$ Periodontol, 73(4), 392- 400.

Demircan, N., Gurel, A., Armutcu, F., Unalacak, M., Aktunc, E., \& Atmaca, H. (2008). The evaluation of serum cystatin $\mathrm{C}$, malondialdehyde, and total antioxidant status in patients with metabolic syndrome. Med Sci Monit, 14(2), CR97-101. Retrieved from https://www.ncbi.nlm.nih.gov/pubmed/18227768

Desmond Jay, Hirofumi Hitomi, \& Griendling, K. K. (2006). Oxidative stress and diabetic cardiovascular complications. Free Radical Biology \& Medicine 40, 40, 183- 192. doi:10.1016/j.freeradbiomed.2005.06.018

Gallou, G., Ruelland, A., Legras, B., Maugendre, D., Allannic, H., \& Cloarec, L. (1993). Plasma malondialdehyde in type 1 and type 2 diabetic patients. Clinica chimica acta, 214(2), 227-234.

Hoang, H. H., Padgham, S. V., \& Meininger, C. J. (2013). Larginine, tetrahydrobiopterin, nitric oxide and diabetes. Current Opinion in Clinical Nutrition \& Metabolic Care, 16(1), 76-82.

Indurkar, M. S., Maurya, A. S., \& Indurkar, S. (2016). Oral Manifestations of Diabetes. Clinical diabetes, 34, 54-57.

Kadir, T., Pisiriciler, R., Akyüz, S., Yarat, A., Emekli, N., \& Ipbüker, A. (2002). Mycological and cytological examination of oral candidal carriage in diabetic patients and non-diabetic control subjects: Thorough analysis of local aetiologic and systemic factors. J Oral Rehabil., 29, 452-457.

Kosoko, A. M., Olayanju, O. A., Rahamon, S. K., \& Arinola, O. G. (2017). Salivary lipid peroxidation and antioxidant status in Nigerian cigarette smokers with or without periodontitis. Asian Journal of Medicine and Health, 3(3), 1-9. doi:10.9734/AJMAH/2017/30749

Leoncini, G., Signorello, M. G., Piana, A., Carrubba, M., \& U, A. (1997). Hyperactivity and increased hydrogen peroxide formation in platelets of NIDDM patients. Thrombosis Research, 86(2), 153- 160.

Maciejczyk, M., Zalewska, A., \& Ladny, J. R. (2019). Salivary antioxidant barrier, redox status, and oxidative damage to proteins and lipids in healthy children, adults and the elderly. oxidative medicine and cellular longevity, 2019, $1-12$.

Maritim, A. C., Sanders, R. A., \& Watkins, J. B., 3rd. (2003). Diabetes, oxidative stress, and antioxidants: a review. $J$ Biochem Mol Toxicol, 17(1), 24-38. doi: $10.1002 /$ jbt. 10058

Nakhjavani, M., Esteghamati, A., Nowroozi, S., Asgarani, F., Rashidi, A., \& Khalilzadeh, O. (2010). Type 2 diabetes mellitus duration: an independent predictor of serum malondialdehyde levels. Singapore medical journal, $51(7), 582$.
Papanicolaou, D. A., Mullen, N., Kyrou, I., \& Nieman, L. K. (2002). Nighttime salivary cortisol: a useful test for the diagnosis of cushing's syndrome. $J$ Clin Endocrinol Metab, 87, 4515- 4521.

Paquette, D. W., \& Williams, R. C. (2000). Modulation of host inflammatory mediators as a treatment strategy for periodontal diseases. Periodontol, 24(1), 239- 252

Pitocco, D., Tesauro, M., Alessandro, R., Ghirlanda, G., \& Cardillo, C. (2013). Oxidative Stress in Diabetes Implications for Vascular and Other Complications. Int. J. Mol. Sci., 14, 21525-21550. doi:10.3390/ijms141121525

Pitocco, D., Zaccardi, F., Di Stasio, E., Romitelli, F., Santini, S. A., Zuppi, C., \& Ghirlanda, G. (2010). Oxidative stress, nitric oxide, and diabetes. The review of diabetic studies: $R D S, 7(1), 15$.

Rehman, K., \& Akash, M. S. H. (2017). Mechanism of Generation of Oxidative Stress and Pathophysiology of Type 2 Diabetes Mellitus: How Are They Interlinked? J Cell Biochem, 118(11), 3577-3585. doi:10.1002/jcb.26097

Slatter, D. A., C.H. Bolton, C. H., \& Bailey, A. J. (2000). The importance of lipid-derived malondialdehude in diabetes mellitus. Diabetologia, 43, 550- 557.

Sunitha, M., \& Shanmugam, S. (2006). Evaluation of salivary nitric oxide levels in oral mucosal diseases: a controlled clinical trial Indian J Dent Res, 17, 117-120.

Takeichi, O., Hayashi, M., Tsurumachi, T., Tomita, T., Ogihara, H., Ogiso, B., \& Saito, T. (1999). Inducible nitric oxide synthase activity by interferon-gamma-producing cells in human radicular cysts. Int Endod J 1999; 32(2): 124-30. Int Endod J, 32(2), 124- 130.

Thomas, B., Ramesh, A., Suresh, S., \& Prasad, B. R. (2013). A comparative evaluation of antioxidant enzymes and selenium in the serum of periodontitis patients with diabetes mellitus type 2. Contemp Clin Dent, 4, 176-180.

Trivedi, S., Lal, N., Mahdi, A. A., Mittal, M., Singh, B., \& Pandey, S. (2014). Evaluation of antioxidant enzymes activity and malondialdehyde levels in patients with chronic periodontitis and diabetes mellitus. Journal of periodontology, 85(5), 713-720.

Zygula, A., Kosinski, P., Zwierzchowska, A., Sochacka, M., Wroczynski, P., Makarewicz-Wujec, M. Giebultowicz, J. (2019). Oxidative stress markers in saliva and plasma differ between diet-controlled and insulin-controlled gestational diabetes mellitus. Diabetes Res Clin Pract, 148, 72-80. doi:10.1016/j.diabres.2018.11.021 